\title{
Agôn
}

Revue des arts de la scène

HS 2 | 2014

Mettre en scène le conte

\section{La place des contes dans les programmes scolaires}

\section{Roxane Paillier}

\section{(2) OpenEdition}

\section{Journals}

Édition électronique

URL : http://journals.openedition.org/agon/3144

DOI : $10.4000 /$ agon.3144

ISSN : 1961-8581

\section{Éditeur}

Association Agôn

\section{Référence électronique}

Roxane Paillier, «La place des contes dans les programmes scolaires », Agôn [En ligne], HS 2 | 2014, mis en ligne le 04 mars 2015, consulté le 10 décembre 2020. URL : http://journals.openedition.org/ agon/3144; DOI : https://doi.org/10.4000/agon.3144

Ce document a été généré automatiquement le 10 décembre 2020.

Association Agôn et les auteurs des articles 


\title{
La place des contes dans les programmes scolaires
}

\author{
Roxane Paillier
}

Les contes ont longtemps été transmis oralement. Au milieu du xvi siècle, Straparola livre les premières transcriptions littéraires de contes populaires vénitiens. En 1625, le napolitain Basile rédige Lo Cunto de li cunti (Le Conte des contes regroupant cinquante contes de fées), plus communément désigné Pentamerone. Le genre du conte de fées est né en France au XVII ${ }^{\mathrm{e}}$ siècle, et en marge du conte merveilleux de Charles Perrault et du "Cercle des conteuses ${ }^{1}$ (Mademoiselle Lhéritier, nièce de Perrault, Madame d'Aulnoy ou encore Mademoiselle de La Force), se développent des sous-catégories répondant aux préoccupations de l'époque : contes philosophiques, moraux et même libertins. Les contes sont aussi bien appréciés dans les milieux populaires que dans les milieux lettrés.

2 A partir du xviII siècle, ils sont largement diffusés grâce au développement de la littérature de colportage. La majorité des textes sont publiés par la Bibliothèque bleue. De 1704 à 1717, Antoine Galland traduit et publie plusieurs volumes des contes arabes Les Mille et Une Nuits. Il transmet une culture étrangère tout en prenant des libertés avec le manuscrit qu'il a en sa possession : sa traduction, considérée comme une vulgate, est un succès en France; Georges May considère la traduction d'Antoine Galland comme une œuvre $^{2}$. En 1757, Madame Leprince de Beaumont, éducatrice de jeunes aristocrates anglaises, propose une version simplifiée de La Belle et la Bête destinée au jeune public dans une intention didactique et morale (le conte est diffusé pour la première fois en France par Madame de Villeneuve en 1740).

3 A partir de la seconde moitié du XIX $x^{e}$ siècle, les contes se démocratisent et connaissent de nombreuses adaptations, notamment musicales. L'édition en plein essor, la naissance de la littérature de jeunesse et l'intérêt croissant porté à l'image ainsi que le développement de la scolarisation favorisent leur diffusion. Les contes de Charles Perrault sont illustrés par Gustave Doré et dès la fin du XIX $x^{e}$ siècle, les vertus pédagogiques de l'image sont confirmées par l'éducation nationale française ${ }^{3}$.

Depuis le milieu $d u x^{e}$ siècle, les contes se déclinent sur une multitude de supports: albums, films d'auteur, films d'animation, œuvres musicales et adaptations théâtrales. Les 
auteurs de comédies musicales, séries télévisées et même de mangas se sont emparés récemment des contes. Si les contes sont étudiés à l'école pour leur aspect littéraire et leur moralité, cette polyexploitation des contes offre de nouvelles potentialités didactiques. L'inscription de Cendrillon ${ }^{4}$, réécriture théâtrale de Joël Pommerat, au programme limitatif du Baccalauréat Littéraire théâtre (épreuve obligatoire), nous donne l'occasion d'interroger la place des contes dans les programmes scolaires ainsi que les déplacements opérés dans leurs usages didactiques.

\section{Usages didactiques du conte à l'école et au collège}

5 Les contes à l'école élémentaire et à l'école primaire constituent une référence littéraire au même titre que les fables, les œuvres poétiques et théâtrales. Ainsi, les contes sont inscrits dans les listes de référence 2013 du ministère de l'Education nationale (cycles 2 et 3), La Littérature à l'école ${ }^{5}$, comme des supports permettant aux élèves de se constituer une première culture littéraire partagée.

Durant ces premières années de formation, le recours aux contes est à relier à différents objectifs pédagogiques : la lecture et l'oralité qui vont favoriser le développement de l'imaginaire, puis l'écriture à l'école primaire. L'album est un support privilégié pour stimuler à la fois les capacités réflexives, l'imaginaire et la créativité de l'enfant ${ }^{6}$ dont la personnalité, les facultés cognitives et herméneutiques sont en pleine construction.

Par sa dimension morale et manichéenne, le conte de fées est considéré comme propre à transmettre des valeurs. En effet, Charles Perrault revendique la dimension instructive et morale de ses contes dans sa préface. Le conte, comme la fable, divertit et instruit. Sa fonction éducative peut prendre des formes diverses: délivrer une leçon de vie fondée sur des valeurs morales, alimenter l'imagination, expliquer une particularité du monde tout en permettant aux enfants de s'identifier aux personnages types dès leur plus jeune âge. Nous tenons à souligner que le plaisir est ce qui a fait le succès de ce genre mondain. Lorsque la morale est invoquée, elle apparaît comme un alibi. Pour André Jolles, les héros des contes sont des produits de «dispositions mentales déterminées $»^{7}$. Ainsi, le conte véhicule une « morale naïve » qui n'est pas apte à servir un prêche en bonne et due forme à l'instar des personnages ambigus comme le Chat botté utilisant la ruse pour offrir une vie confortable à son maître mal né.

Dans son ouvrage Les contes à l'école ${ }^{8}$ paru en 1997, Serge Martin souligne la multiplicité des entrées possibles et rejette la lecture des contes par le prisme formaliste du schéma narratif qui selon lui est réducteur. Car le conte permet de nombreuses approches : par la lecture (la transmission orale est assurée par les professeurs mais également par les conteurs lors de "L'Heure du conte » par exemple), par la littérature jeunesse par le biais des albums et le recours à l'image ou encore par une approche comparative. Des déplacements peuvent également être opérés dans les usages didactiques du conte, qu'il s'agisse des contes « détournés » (réécritures, transpositions et parodies) ${ }^{9}$ ou de contes mis en scène (adaptations théâtrales). Les compétences littéraires visées (linguistique, encyclopédique, logique et rhétorique) demeurent inchangées mais le détournement des contes place le lecteur en situation problème. Par conséquent, l'approche sera plus ludique et davantage centrée sur le rôle des stéréotypes.

Dans son ouvrage sur les contes détournés, Christiane Connan-Pintado définit le conte à la fois comme un "genre littéraire" et un "genre scolaire, objet et outil d'apprentissage $»^{10}$. Elle engage les enseignants à mettre en relation les œuvres et à lire 
des contes détournés à condition de bien authentifier le conte source au préalable. Ainsi, elle encourage la lecture en réseaux qui permet de rapprocher des œuvres et de former un lecteur cultivé. Il est important de souligner que depuis 2002, les programmes scolaires insistent sur la dimension intertextuelle de la littérature de jeunesse. Afin que l'apprentissage soit cohérent, l'élève doit être capable d'identifier le conte source à l'origine des réécritures.

Le conte mis en scène est une autre des formes de réécriture qui peut être étudié à partir du CM1. Bruno Castan se consacre par exemple depuis 1971 à l'écriture théâtrale pour la jeunesse $^{11}$. Sa pièce Belle des eaux ${ }^{12}$, réécriture de la version de Madame Leprince de Beaumont de La Belle et la Bête, a été sélectionnée en 2004, 2007 et 2013 par l'Education nationale pour le cycle 3 du primaire et pour la liste de « Lectures pour les collégiens » en $2013^{13}$. Comme pour Cendrillon de Joël Pommerat, ces inscriptions dans les listes officielles permettent une ouverture des pratiques pédagogiques autour des contes de l'école primaire au lycée. L'Education nationale incite ainsi les enseignants à tenir compte de la grande plasticité des contes dans leurs usages.

6 Au collège, les contes et récits merveilleux sont inscrits au programme de sixième ${ }^{14}$. Les élèves doivent lire au moins deux contes choisis par leur professeur parmi la liste proposée par le programme. Plusieurs objectifs pédagogiques sont visés : l'étude de la langue et de la structure du conte, la lecture analytique, la lecture de l'image et l'expression écrite. Le conte n'est pas seulement appréhendé comme un genre littéraire, il est également convoqué pour servir de support à l'apprentissage de la grammaire ou comme point de départ d'une expression écrite.

Dans les supports pédagogiques ${ }^{15}$ destinés aux élèves, les exercices sont consacrés essentiellement à la compréhension. Dans le manuel, trois types de conte sont proposés : le conte à moralité, le conte oriental et le récit d'apprentissage. Les questions de compréhension portent sur la structure, le schéma narratif et actantiel (les forces agissantes). Dans le cahier d'activités, les questions sont du même ordre : personnages (allié/ennemi), épreuves et moralité. A la différence du manuel, elles sont présentées de manière ludique : quiz vrai/faux, propositions à relier, jeu de l'intrus. De manière plus scolaire, des questions ouvertes ainsi que des travaux guidés d'écriture sont également proposés. Qu'il s'agisse de tirer une leçon d'un conte ou de rédiger une morale à la manière de Perrault, la moralité reste un élément d'analyse dominant. Enfin, les contes parodiques sont également présents. Par exemple, à partir du Petit Chaperon rouge, un travail d'écriture d'inversion des rôles est proposé aux élèves. Nous relevons d'ailleurs que dans la pièce de Joël Pommerat ${ }^{16}$, la morale s'adresse au loup et non à la fillette.

Dans le cadre de cette étude, de nombreuses activités pédagogiques peuvent être exploitées par l'enseignant. Par exemple, écrire un conte sur le site de la $\mathrm{BnF}^{17}$ permet de travailler à la fois l'expression écrite tout en formant les élèves à l'utilisation des TIC (Technologies de l'Information et de la Communication). De même, le professeur est libre de prolonger la lecture d'un conte en faisant entrer en correspondance différentes œuvres artistiques. La thématique « Arts, mythes et religions » d'histoire des arts en offre la possibilité. Un travail peut être proposé autour des adaptations cinématographiques et théâtrales, des œuvres musicales et des illustrations (ces dernières permettent d'initier les élèves à l'étude de l'image). Cette partie de la séquence peut être menée en partenariat avec le professeur documentaliste qui peut proposer différents supports : contes illustrés, albums de littérature jeunesse et documents audiovisuels. En travaillant en interdisciplinarité et en insistant sur les mises en réseaux, les enseignants amènent les 
élèves à prendre conscience de l'influence du conte et de sa transposition dans les autres arts.

7 Nous avons pu observer une certaine continuité dans l'étude des contes. Les actants, les actions et la moralité restent les principaux éléments analysés. Cependant, nous pouvons relever une ouverture des pratiques pédagogiques des enseignants qui se tournent vers les arts : arts du visuel et du spectacle vivant comme le montre l'inscription de Cendrillon de Joël Pommerat au Baccalauréat Littéraire théâtre. Les différentes options, le développement du travail en interdisciplinarité ainsi que l'enseignement de l'histoire des arts contribuent pleinement à cette ouverture.

\section{Les contes merveilleux au lycée général et technologique : un enseignement optionnel}

8 A la différence des programmes obligatoires du lycée général et technologique, le programme du Baccalauréat professionnel ${ }^{18}$ s'appuie sur les contes. Nous pouvons dès lors nous demander si ces différents traitements entre Baccalauréat général et technologique et Baccalauréat professionnel n'induisent pas une hiérarchie implicite. En effet, le conte peut être considéré comme étant plus accessible aux élèves choisissant la voie professionnelle.

En classe de seconde professionnelle, les élèves étudient les parcours de personnages et plus particulièrement les héros littéraires, l'évolution d'un personnage, les valeurs qu'il incarne et son impact sur le lecteur. Nous soulignons que les contes traditionnels n'ont pas affaire à des personnages, mais à des agents dénués de psychologie et supports d'actions. La personnalisation des récits s'est accentuée au fil du temps, par contamination d'autres types de récits à succès tels que le roman d'aventures et le romanfeuilleton au XIX ${ }^{e}$ siècle. Il est intéressant de relever que ces agents sont traités comme des personnages denses dans ce programme.

En classe de première, un objet d'étude est consacré à l'imaginaire. Il s'agit ici de s'interroger sur la fonction et le statut des contes, des fables et autres récits imaginaires. Les contes ne sont pas à réduire aux seuls contes merveilleux (contes d'animaux, contes religieux, contes facétieux ou encore contes de tous les pays), mais il est manifeste que le succès des contes de fées a occulté les autres types de contes, très vivaces dans d'autres cultures. Enfin, l'enseignant s'intéresse également à la réception de ces œuvres et à leur lectorat. Nous pouvons constater que le conte n'est pas appréhendé en tant que genre littéraire.

9 Les contes et récits merveilleux n'apparaissent pas dans les programmes obligatoires du lycée général et technologique. En revanche, le conte philosophique entre dans le corpus du quatrième objet d'étude "Genres et formes de l'argumentation : XVII et XVIII ${ }^{\mathrm{e}}$ siècle " de la classe de seconde générale et technologique ${ }^{19}$ ainsi que dans le quatrième objet d'étude commun aux classes de première des séries générales : « La question de l'Homme dans les genres de l'argumentation du XVI ${ }^{\mathrm{e}}$ à nos jours ».

L'objet d'étude spécifique pour l'enseignement de littérature en première Littéraire, « Les réécritures, du XVII siècle jusqu'à nos jours ", peut convoquer l'étude des contes puisqu'il engage à aborder « les questions de genre, de registre et d'intertextualité » et à travailler "sur les phénomènes de citation, d'imitation, de variation et de transposition ${ }^{20}$ ». Ainsi, l'enseignant peut proposer des œuvres appartenant à des genres différents et aborder la 
notion de transposition ou partir d'un type de personnage et observer ses variations. Ces études peuvent être approfondies dans le cadre de l'histoire des arts en ouvrant le corpus à d'autres œuvres (peinture, arts visuels, cinéma et opéra). Nous soulignons que le professeur est libre dans le choix des œuvres pour bâtir son corpus.

Les contes peuvent être abordés dans les dispositifs particuliers du lycée tels que l'enseignement d'exploration "Littérature et société » en classe de seconde générale et technologique ou lors des TPE (Travaux Personnels Encadrés) en première Littéraire. En effet, pour l'enseignement Littérature et Société, le domaine d'exploration «Images et langages: donner à voir, se faire entendre " offre comme point d'entrée possible les œuvres littéraires et adaptations cinématographiques ${ }^{21}$. Ainsi, une étude sur la transposition du conte au cinéma peut être proposée dans ce cadre (films et films d'animation). Comme pour l'enseignement en première Littéraire, il s'agit de pistes proposées dans les programmes. L'enseignant est libre dans le choix des œuvres (le conte est une suggestion parmi d'autres).

En ce qui concerne les TPE, épreuve anticipée du Baccalauréat, les deux thèmes spécifiques de la série littéraire "Héros et personnages" et "Représentations et réalités » offrent la possibilité aux groupes de s'appuyer sur un corpus de contes. Pour le premier, une étude peut être menée sur les personnages types ou les itinéraires héroïques, leur rayonnement et leurs réécritures ${ }^{22}$. Le deuxième thème permet quant à lui de travailler sur la construction du personnage ${ }^{23}$. Nous précisons qu'il s'agit de pistes : les élèves sont guidés par les enseignants mais libres de choisir un sujet qui entre dans les thèmes proposés par le Ministère de l'Education nationale. Il n'existe pas de statistiques sur les sujets traités par les élèves au niveau national. En tant que co-encadrante de l'enseignement Littérature et Société depuis 2011 dans un lycée catholique privé sous contrat d'association avec l'Etat, j'ai constaté que les élèves ne se tournent pas spontanément vers les contes. Lorsque les élèves travaillent en groupe sur un sujet de leur choix pour préparer un exposé, ils privilégient les sujets sur les médias, les réseaux sociaux, les livres numériques, la censure ou la propagande. En moyenne, sur une quinzaine de groupe à l'année, un seul travaille sur les contes et leurs adaptations cinématographiques. Il est intéressant de noter que le choix de ce sujet concerne un public féminin. De même, je n'ai pas relevé dans mon établissement de sujet de TPE en lien avec les contes en série littéraire.

11 En classe de première Economique et Sociale, en Sciences Economiques et Sociales et plus précisément en sociologie générale ${ }^{24}$, le conte peut constituer une entrée possible pour comprendre les processus de socialisation (socialisation de l'enfant : construction de la personnalité de l'enfant par intériorisation de valeurs et construction sociale des rôles associés au sexe). Le conte est convoqué pour faire prendre conscience aux élèves des valeurs qu'il véhicule : la représentation du bien et du mal, les valeurs morales telles que la justice ou l'honnêteté, les valeurs familiales et sociales. Si les valeurs individuelles peuvent entrer en conflit avec les valeurs collectives, François Flahault, philosophe et anthropologue dont les essais ont influencé Joël Pommerat, souligne l'importance des relations dans la construction de l'identité. Pour le philosophe, le conte est un lieu de représentation de l'identité qui illustre la réalisation de soi grâce à sa relation avec les autres. François Flahault pense l'identité comme relationnelle et évolutive. En ce sens, les contes posent la question de la relation aux autres et de la manière dont se construit un espace où l'on est plusieurs ${ }^{25}$. Cet exemple d'utilisation sociologique, voire philosophique, du conte le sort du strict usage littéraire. 
12 Il est important de mentionner le programme de Terminale Littéraire des sessions 2006-2007 et 2007-2008, les Contes de Charles Perrault illustrés par Gustave Doré (programme de littérature : Domaine langage verbal et images - Littérature et langage de l'image) ${ }^{26}$. Il s'agissait de s'intéresser à l'illustration et aux choix opérés par l'illustrateur pour créer sa propre œuvre à partir de contes sources. Nous précisons que ce domaine a été inscrit au programme du Baccalauréat Littéraire durant deux années et que la liste des œuvres de cet enseignement est régulièrement renouvelée.

Pour l'année scolaire 2014-2015 et la session 2015 du baccalauréat, le programme limitatif de l'enseignement de spécialité théâtre de la série L (épreuve obligatoire) comprend le texte Cendrillon de Joël Pommerat ${ }^{27}$. Cette inscription de Cendrillon, conte réécrit et mis en scène, au baccalauréat théâtre implique une autre spécificité des usages du conte dans les programmes du lycée puisque cette transposition théâtrale implique un déplacement générique, le passage du raconté au représenté. Elle permet de s'interroger sur l'intermédialité et les transformations nécessaires pour adapter un conte sur scène. Les élèves travaillent sur les processus de création : il est intéressant de souligner que pour Joël Pommerat, l'écriture et la mise en scène se pensent ensemble ${ }^{28}$. Patrick Laudet, Inspecteur général chargé du Théâtre, souligne l'intérêt du travail de Joël Pommerat, " écrivain de plateau », dont l'enjeu est de sortir de la dualité auteur/metteur en scène ${ }^{29}$. Si la pièce pose des questions très actuelles liées à des thèmes universels (le deuil, la différence, l'isolement, la relation de couple, l'amour), elle est également très riche pour aborder les enjeux de la scène. L'étude de cette réécriture doit amener les élèves à " formuler des interprétations réfléchies et argumentées " ${ }^{30}$ sur l'œuvre achevée.

Nous pouvons constater que depuis la fin des années 1990, les contes ne sont plus étudiés seuls. Ces textes patrimoniaux permettent une lecture en réseaux. La polyexploitation des contes, qui se déclinent sur différents supports, permet d'aborder les notions de genre littéraire, d'intermédialité et de transmédialité.

\section{L'étude des contes dans le supérieur : approches littéraire, psychanalytique et didactique}

Les contes sont abordés dans les programmes d'histoire littéraire et culturelle dans les études de Lettres ou dans les cursus spécifiques. Par exemple, le master LIJE (Lettres, Littératures et Civilisation Spécialité Littérature de Jeunesse) proposé au Mans comporte un cours sur les contes en Master 1. Chaque année, de nombreux sujets de mémoire portant sur les albums de contes sont soutenus.

16 L'empreinte structuraliste dominante dans les années 1970 est en passe de s'effacer. La perspective théorique et méthodologique s'en trouve modifiée. Le conte devient un exemple privilégié pour illustrer des problématiques actuelles s'interrogeant sur le canon littéraire et le cloisonnement des genres et des disciplines : il peut ainsi être évoqué à la faveur d'enseignements sur les échanges entre les littératures orales et la création littéraire écrite; sur les circulations entre Orient et Occident; sur les codes et les stéréotypes littéraires; sur l'écriture, la réécriture, la variance; ou encore sur la complémentarité entre littérature et psychanalyse.

17 En 1976, Bruno Bettelheim publie Psychanalyse des contes de fées ${ }^{31}$. L'ouvrage de Bettelheim est devenu un classique de l'approche psychanalytique des contes. Selon lui, ces derniers reflètent des angoisses et des conflits propres à l'enfance. En plus de leur fonction 
divertissante, les contes aident l'enfant à mieux cerner ses émotions et assurent une fonction thérapeutique. Bruno Bettelheim s'est également penché sur l'art de raconter les contes de fées, expérience de plaisir partagé entre le conteur et son auditoire. Cette approche offre pour les enseignants une alternative à l'approche structuraliste qui peut se révéler appauvrissante.

Les enseignants n'étudient pas directement ces interprétations symboliques dans le primaire et le secondaire, même si les élèves ont souvent conscience de ces conflits. A ce stade, l'analyse reste essentiellement centrée sur la dimension manichéenne. En effet, l'œuvre de Bettelheim s'inscrit dans les cursus universitaires de Psychologie, Lettres modernes et Enseignement. La culture philosophique enseignée en classe de terminale et tout particulièrement la conception freudienne de l'inconscient - est une propédeutique à l'étude de Psychanalyse des contes de fées.

Les masters MEEF (Métiers de l'Enseignement, de l'Education et de la Formation) créés en 2013 pour le premier et second degré ou autre spécialisation, dispensent des éléments de culture disciplinaire et didactique pour les futurs enseignants. C'est dans ce cadre que sont enseignées les approches théoriques et pratiques des contes ainsi que les connaissances en littérature de jeunesse.

19 En ce qui concerne le conte mis en scène, l'adaptation cinématographique, la musique ou encore les arts visuels, il est évident que les cursus de théâtre, de cinéma, de musicologie ou d'art dans le supérieur peuvent convoquer les contes dans leurs apprentissages.

\section{Vers une ouverture des usages du conte dans les programmes scolaires}

20 Si les contes sont inscrits au programme des cycles 2 et 3 de l'école et en classe de sixième, leur étude reste assez inégale selon les filières et les séries du secondaire. Par rapport aux autres genres littéraires, le conte merveilleux est sous-exploité : il n'est pas présent dans les programmes obligatoires du lycée général et technologique.

21 L'étude de la structure du récit, l'analyse des motifs et de la morale restent les éléments d'analyse dominants en sixième. Mais le conte ne sert pas seulement à enseigner des modèles de comportements, l'étude de ses détournements dès l'école primaire ainsi que sa lecture sociologique en première Economique et Sociale engagent une réflexion sur les valeurs, les stéréotypes et les constructions sociales qu'il induit. Comme le montre l'inscription de Cendrillon, réécriture théâtrale de Joël Pommerat, à l'épreuve obligatoire du Baccalauréat Littéraire théâtre, nous relevons toutefois une ouverture des usages des contes dans les programmes scolaires littéraires et artistiques. En effet, le conte n'est plus seulement abordé en tant que genre littéraire mais aussi pour les diverses exploitations possibles qu'il offre. Cela est en partie dû à ses déclinaisons sur différents supports et à sa grande plasticité. L'enseignant, laissé libre de ses choix, dispose d'un corpus pléthorique et de plusieurs points d'entrée: il veille à la cohérence des approches et supports proposés aux élèves. 


\section{NOTES}

1. Voir Contes, Mademoiselle Lhéritier, Mademoiselle Bernard, Mademoiselle de La Force, Madame Durand, Madame d'Auneuil, édition critique établie par Raymonde Robert, Paris, Honoré Champion, Bibliothèque des Génies et des Fées, 2005.

2. MAY, Georges, Les Mille et une nuits d'Antoine Galland ou Le chef-d'œuvre invisible, Paris, PUF, 1986.

3. «Voir, c'est déjà savoir " affirme un responsable de l'éducation nationale. Voir BOURDIER, Philippe et CAGLAR, Pascal, Les Contes de Charles Perrault illustrés par Gustave Doré, Paris, Ellipses, Réseau diagonales, 2006, p. 102.

4. POMMERAT, Joël, Cendrillon, Arles, Actes Sud, Babel, 2013.

5. Ministère de l'Education nationale et Eduscol, La Littérature à l'école, listes de référence (cycle 2 et cycle 3), MEN-DGESCO, 2013, URL : http://eduscol.education.fr/cid50485/litterature.html - lien0 6. Voir les travaux d'Henri Wallon et de Donald Winnicott.

7. JOLLES, André, Formes simples, Paris, Seuil, 1972, p. 34.

8. MARTIN, Serge, Les contes à l'école, Paris, Bertrand Lacoste, Parcours didactiques à l'école, 1997.

9. Ibid., « Découvrir la parodie (des contes: des écritures) », p. 110-116 et CONNAN-PINTADO, Christiane, Lire des contes détournés à l'école. A partir des contes de Perrault de la GS au CM2, Paris, Hatier, Hatier pédagogie, 2009.

10. Ibid., p. 5.

11. Voir le site des Editions Théâtrales Jeunesse où sont publiées ses œuvres, URL: http:// www.editionstheatrales.fr/collections/theatrales-jeunesse-4.html

12. CASTAN, Bruno, Belle des eaux, Montreuil, Editions Théâtrales, Théâtrales Jeunesse, 2002.

13. Ministère de l'Education nationale et Eduscol, Liste de "Lectures pour les collégiens ", MEN, 2013, URL : http://eduscol.education.fr/cid83185/liste-litterature-pour-les-collegiens.html

14. Ministère de l'Education nationale-DGESCO, Programmes français (classes de sixième, cinquième, quatrième, troisième), CNDP, mars 2014, URL: http://www2.cndp.fr/archivage/ valid/140235/140235-18635-24218.pdf

15. Nous prenons pour référence le cahier d'activités et le manuel suivants : CARPENTIER, claude (dir.), Mon cahier de lecture et d'écriture. Français $6^{e}$, Paris, Bordas, Les cahiers de français, 2014 et POTELET, Hélène (dir.), Français. Livre unique 6 ${ }^{e}$, Paris, Hatier, Rives bleues, 2009.

16. POMMERAT, Joël (auteur) et LERAY, Marjolaine (ill.), Le Petit chaperon rouge, Arles, Actes SudPapiers, Heyoka Jeunesse, 2005.

17. Voir l'atelier d'écriture proposé par la BnF, URL : http://expositions.bnf.fr/contes/pedago/ atelier/index.htm

18. Ministère de l'Education nationale-DGESCO, Programmes Français, Histoire, géographie et éducation civique (Baccalauréat professionnel), CNDP, septembre 2010, URL : http://www2.cndp.fr/ archivage/valid/149705/149705-20928-26839.pdf

19. Ministère de l'Education nationale, de la Jeunesse et de la Vie associative-DGESCO, Programmes Français Littérature (classes de seconde générale et technologique, classe de première des séries générales), CNDP, avril 2011, URL: http://www2.cndp.fr/archivage/ valid/154252/154252-22107-28114.pdf

20. Idem, p. 22.

21. Ministère de l'Education nationale. Bulletin officiel spécial $n^{\circ} 4$ du 29 avril 2010: Programme de Littérature et société en classe de seconde générale et technologique - Enseignement d'exploration, Ministère de l'Education nationale, 2010, p.4-5, URL: http://media.education.gouv.fr/file/ special_4/74/5/litterature_societe_143745.pdf 
22. Ministère de l'Education nationale-DGESCO, Ressources pour la classe de première générale Série littéraire (L), Travaux Personnels encadrés, Thème spécifique Héros et personnages, Eduscol, juillet 2011, URL : $\quad$ http://cache.media.eduscol.education.fr/file/TPE/40/9/ LyceeGT_TPE_L_1_Heros_Personnages_186409.pdf

23. Ministère de l'Education nationale-DGESCO, Ressources pour la classe de première générale Série littéraire (L), Travaux Personnels encadrés, Thème spécifique Représentations et réalités, Eduscol, juillet 2011, URL : http://cache.media.eduscol.education.fr/file/TPE/41/1/ LyceeGT_TPE_L_1_Representations_Realites_186411.pdf

24. Ministère de l'Education nationale, de la Jeunesse et de la Vie associative-DGESCO, Sciences économiques et sociales (classe de première de la série ES), CNDP, avril 2011, URL: http:// www2.cndp.fr/archivage/valid/154250/154250-22097-28104.pdf

25. Voir le site de François Flahault, François Flahault, Philosophe et anthropologue, 2014, URL: http://www.francoisflahault.fr/

26. Ministère de l'Education nationale, Bulletin officiel spécial $n^{\circ} 17$ du 27 avril 2006: Baccalauréat: Programme de littérature de la classe terminale littéraire - année scolaire 2006-2007, MEN, 2006, URL : http://www.education.gouv.fr/bo/2006/17/MENE0601125N.htm

27. Ministère de l'Education nationale, Bulletin officiel $n^{\circ} 8$ du 20 février 2014 : enseignements primaire et secondaire, Baccalauréat, MEN, 2014, URL: http://www.education.gouv.fr/pid25535/ bulletin_officiel.html ?cid_bo $=77026$

28. Aux nouvelles écritures théâtrales (Aneth), Dossiers auteurs : Joël Pommerat, Aneth, [s. d.], URL : http://www.aneth.net/doc_auteur_art.php ?IdAuteur=639\&IdDocument =170

29. Théâtre Odéon, Nouer un dialogue avec l'enfance, Entretien avec Patrick Laudet, Théâtre Odéon, 2013, URL : http://www.theatre-odeon.eu/fr/nouer-un-dialogue-avec-l-enfance

30. Ministère de l'Education nationale, de la jeunesse et de la vie associative-DGESCO, Arts (classes de première et terminale des séries générales et technologiques), CNDP, avril 2011, URL: http:// www2.cndp.fr/archivage/valid/154251/154251-22101-28108.pdf

31. BETTELHEIM, Bruno, The Uses of Enchantment: The Meaning and Importance of Fairy Tales, London, Thames and Hudson, 1976. BETTELHEIM, Bruno, Psychanalyse des contes de fées (trad. de l'américain par Théo Carlier), Paris, Hachette Littératures, Pluriel, 1998. 